\title{
Kamenev-type oscillation criteria for higher-order nonlinear dynamic equations on time scales
}

\author{
Xin $\mathrm{Wu}^{1}$, Taixiang Sun ${ }^{1 *}$, Hongjian $\mathrm{Xi}^{2}$ and Changhong Chen ${ }^{1}$
}

\section{"Correspondence:}

stx1963@163.com

${ }^{1}$ College of Mathematics and

Information Science, Guangxi

University, Nanning, Guangxi

530004, P.R. China

Full list of author information is

available at the end of the article

\begin{abstract}
In this paper, we investigate the oscillation of the following higher-order dynamic equation:

$$
\left\{r_{n}(t)\left[\left(r_{n-1}(t)\left(\cdots\left(r_{1}(t) x^{\Delta}(t)\right)^{\Delta} \cdots\right)^{\Delta}\right)^{\Delta}\right]^{\gamma}\right\}^{\Delta}+F(t, x(\tau(t)))=0
$$

on an arbitrary time scale $\mathbf{T}$, where $n \geq 2, \frac{1}{r_{k}(t)}(1 \leq k \leq n)$ are positive rd-continuous functions on $\mathbf{T}$, and $\gamma$ is the quotient of two odd positive integers, $\tau: \mathbf{T} \rightarrow \mathbf{T}$ with $\tau(t)>t$ and $F \in C(\mathbf{T} \times \mathbf{R}, \mathbf{R})$. We give sufficient conditions under which every solution of this equation is either oscillatory or tends to zero.
\end{abstract}

MSC: 34K11;39A10; 39A99

Keywords: oscillation; dynamic equation; time scale

\section{Introduction}

Let $\mathbf{R}$ be the set of all real numbers, and let $\mathbf{T}$ be a time scale (i.e., a closed nonempty subset of $\mathbf{R}$ ) with $\sup \mathbf{T}=\infty$. In this paper, we study Kamenev-type oscillation criteria of solutions of the following higher-order dynamic equation:

$$
\left\{r_{n}(t)\left[\left(r_{n-1}(t)\left(\cdots\left(r_{1}(t) x^{\Delta}(t)\right)^{\Delta} \cdots\right)^{\Delta}\right)^{\Delta}\right]^{\gamma}\right\}^{\Delta}+F(t, x(\tau(t)))=0, \quad t \in\left[t_{0}, \infty\right)_{\mathrm{T}},
$$

where $t_{0} \in \mathbf{T}$ is a constant and $[t, \infty)_{\mathbf{T}}=[t, \infty) \cap \mathbf{T}$ for any $t \in \mathbf{T}$. Throughout this paper, we assume that the following conditions are satisfied:

$\left(\mathrm{H}_{1}\right) \frac{1}{r_{k}(t)} \in C_{\mathrm{rd}}\left(\left[t_{0}, \infty\right)_{\mathrm{T}},(0, \infty)\right)(1 \leq k \leq n)$.

$\left(\mathrm{H}_{2}\right) \gamma$ is the quotient of two odd positive integers.

$\left(\mathrm{H}_{3}\right) \int_{t_{0}}^{\infty} \frac{1}{r_{k}(s)} \Delta s=\int_{t_{0}}^{\infty}\left[\frac{1}{r_{n}(s)}\right]^{\frac{1}{\gamma}} \Delta s=\infty(1 \leq k \leq n-1)$.

$\left(\mathrm{H}_{4}\right) \tau: \mathbf{T} \rightarrow \mathbf{T}$ is a nondecreasing function with $\tau(t)>t$ for any $t \in \mathbf{T}$.

$\left(\mathrm{H}_{5}\right) \quad F \in C(\mathbf{T} \times \mathbf{R}, \mathbf{R})$ and there exists a positive rd-continuous function $q(t)$ defined on $\mathbf{T}$ such that for any $u \neq 0$,

$$
\frac{F(t, u)}{u^{\gamma}} \geq q(t) .
$$

\section{Springer}

(C) 2013 Wu et al: ilicensee Springer. This is an Open Access article distributed under the terms of the Creative Commons Attribution License (http://creativecommons.org/licenses/by/2.0), which permits unrestricted use, distribution, and reproduction in any medium, provided the original work is properly cited. 
Write

$$
S_{k}(t, x(t))= \begin{cases}x(t) & \text { if } k=0, \\ r_{k}(t) S_{k-1}^{\Delta}(t, x(t)) & \text { if } 1 \leq k \leq n-1, \\ r_{n}(t)\left[S_{n-1}^{\Delta}(t, x(t))\right]^{\gamma} & \text { if } k=n .\end{cases}
$$

Then Eq. (1.1) reduces to the equation

$$
S_{n}^{\triangle}(t, x(t))+F(t, x(\tau(t)))=0 .
$$

By a solution of Eq. (1.3) we mean a nontrivial real-valued function $x(t) \in C_{\text {rd }}^{1}\left(\left[T_{x}, \infty\right)_{\mathrm{T}}\right)$ with $T_{x} \geq t_{0}$, which has the property that $S_{k}(t, x(t)) \in C_{\mathrm{rd}}^{1}\left(\left[T_{x}, \infty\right)_{\mathrm{T}}\right)$ for $0 \leq k \leq n$ and satisfies Eq. (1.3) on $\left[T_{x}, \infty\right)_{\mathrm{T}}$, where $C_{\mathrm{rd}}^{1}$ is the space of differentiable functions whose derivative is rd-continuous. The solutions vanishing in some neighborhood of infinity will be excluded from our consideration. A solution $x(t)$ of Eq. (1.3) is said to be oscillatory if it is neither eventually positive nor eventually negative; otherwise, it is called nonoscillatory.

The theory of time scales, which has recently received a lot of attention, was introduced by Stefan Hilger in his $\mathrm{PhD}$ thesis [1] in order to unify continuous and discrete analysis. The cases when a time scale $\mathbf{T}$ is equal to $\mathbf{R}$ or all integers $\mathbf{Z}$ represent the classical theories of differential and difference equations. Many results concerning differential equations carry over quite easily to corresponding results for difference equations, while other results seem to be completely different from their continuous counterparts. The study of dynamic equations on time scales reveals such discrepancies and helps avoid proving results twice - once for differential equations and once again for difference equations. The general idea is to prove a result for a dynamic equation where the domain of the unknown function is a time scale $\mathbf{T}$. In this way results not only related to the set of real numbers or the set of integers but those pertaining to more general time scales are obtained. Therefore, not only can the theory of dynamic equations unify the theories of differential equations and difference equations, but it also extends these classical cases to cases 'in between', e.g., to the so-called $q$-difference equations when $\mathbf{T}=q^{\mathbf{N}_{0}}$, which has important applications in quantum theory (see [2]). In the last years there has been much research activity concerning the oscillation and asymptotic behavior of solutions of some dynamic equations on time scales, and we refer the reader to the papers [3-17] and the references cited therein.

Recently, Wang [18] extended the Hille and Nehari oscillation theorems to the thirdorder dynamic equation

$$
\left(r_{2}(t)\left(\left(r_{1}(t) x^{\triangle}(t)\right)^{\Delta}\right)^{\gamma}\right)^{\Delta}+q(t) f(x(t))=0 .
$$

Erbe et al. in [19-21] considered the third-order dynamic equations

$$
\begin{aligned}
& \left(a(t)\left[r(t) x^{\Delta}(t)\right]^{\triangle}\right)^{\triangle}+p(t) f(x(t))=0, \\
& x^{\Delta \Delta \Delta}(t)+p(t) x(t)=0
\end{aligned}
$$


and

$$
\left(a(t)\left\{\left[r(t) x^{\Delta}(t)\right]^{\Delta}\right\}^{\gamma}\right)^{\Delta}+f(t, x(t))=0,
$$

respectively, and established some sufficient conditions for oscillation.

Hassan [22] studied the third-order dynamic equation

$$
\left(a(t)\left\{\left[r(t) x^{\Delta}(t)\right]^{\Delta}\right\}^{\gamma}\right)^{\Delta}+f(t, x(\tau(t)))=0
$$

and obtained some oscillation criteria, which improved and extended the results that were established in [19-21].

Hassan [23] studied the Kamenev-type oscillation criteria of the second-order dynamic equation

$$
\left(r(t)\left(x^{\Delta}(t)\right)^{\gamma}\right)^{\Delta}+f(t, x(g(t)))=0
$$

and established some new sufficient conditions, which improved some oscillation results for second-order differential and difference equations.

\section{Some auxiliary lemmas}

We shall employ the following lemmas.

Lemma $2.1[24]$ Let $1 \leq m \leq n$. Then:

(1) $\liminf _{t \rightarrow \infty} S_{m}(t, x(t))>0$ implies $\lim _{t \rightarrow \infty} S_{i}(t, x(t))=\infty$ for $0 \leq i \leq m-1$.

(2) $\limsup _{t \rightarrow \infty} S_{m}(t, x(t))<0$ implies $\lim _{t \rightarrow \infty} S_{i}(t, x(t))=-\infty$ for $0 \leq i \leq m-1$.

Lemma 2.2 Suppose that $x(t)$ is an eventually positive solution of Eq. (1.3), then there exist an integer $\ell \in[0, n]$ and $T \in\left[t_{0}, \infty\right)_{\mathrm{T}}$ such that:

(1) $n+\ell$ is even.

(2) $\ell>0$ implies that $S_{i}(t, x(t))>0$ for any $t \geq T$ and $0 \leq i \leq \ell-1$.

(3) $\ell \leq n-1$ implies that $(-1)^{\ell+i} S_{i}(t, x(t))>0$ for any $t \geq T$ and $\ell \leq i \leq n-1$.

Proof Since $x(t)$ is an eventually positive solution of Eq. (1.3), there exists a $t_{1} \geq t_{0}$ such that $x(t)>0$ and $x(\tau(t))>0$ on $\left[t_{1}, \infty\right)_{\mathrm{T}}$. It follows from (1.3) that

$$
S_{n}^{\Delta}(t, x(t))=-F(t, x(\tau(t))) \leq-q(t) x^{\gamma}(\tau(t))<0 \quad \text { for } t \geq t_{1} .
$$

Hence $S_{n}(t, x(t))$ is decreasing on $\left[t_{1}, \infty\right)_{\mathrm{T}}$.

We claim that $S_{n}(t, x(t))>0$ for all $t \in\left[t_{1}, \infty\right)_{\mathrm{T}}$. If not, there exists a $t_{2} \in\left[t_{1}, \infty\right)_{\mathrm{T}}$ such that

$$
S_{n}(t, x(t)) \leq S_{n}\left(t_{2}, x\left(t_{2}\right)\right)<0 \quad \text { for } t \geq t_{2} .
$$

Then we obtain

$$
S_{n-1}(t, x(t)) \leq S_{n-1}\left(t_{2}, x\left(t_{2}\right)\right)+S_{n}^{\frac{1}{\gamma}}\left(t_{2}, x\left(t_{2}\right)\right) \int_{t_{2}}^{t}\left[\frac{1}{r_{n}(s)}\right]^{\frac{1}{\gamma}} \Delta s \rightarrow-\infty \quad(t \rightarrow \infty) .
$$


By Lemma 2.1, we get $\lim _{t \rightarrow \infty} x(t)=-\infty$, which contradicts with $x(t)>0$ eventually. Then $S_{n}(t, x(t))>0$ for all $t \in\left[t_{1}, \infty\right) \mathrm{T}$. This implies that exactly one of the following is true:

(a) $S_{n-1}(t, x(t))<0$ for $t \geq t_{1}$;

$\left(\mathrm{b}_{1}\right)$ There exists a $t_{3} \geq t_{1}$ such that $S_{n-1}(t, x(t)) \geq S_{n-1}\left(t_{3}, x\left(t_{3}\right)\right)>0$ for $t \geq t_{3}$.

If $\left(b_{1}\right)$ holds, then we obtain by Lemma 2.1

$$
\lim _{t \rightarrow \infty} S_{n-2}(t, x(t))=\lim _{t \rightarrow \infty} S_{n-3}(t, x(t))=\cdots=\lim _{t \rightarrow \infty} x(t)=\infty
$$

Thus the conclusions of Lemma 2.2 hold.

If $\left(\mathrm{a}_{1}\right)$ holds, then $S_{n-2}(t, x(t))$ is strictly decreasing on $\left[t_{1}, \infty\right)_{\mathrm{T}}$ and exactly one of the following is true:

( $\left.\mathrm{a}_{2}\right) S_{n-2}(t, x(t))>0$ for $t \geq t_{1}$;

$\left(\mathrm{b}_{2}\right)$ There exists a $t_{4} \geq t_{1}$ such that $S_{n-2}(t, x(t)) \leq S_{n-1}\left(t_{4}, x\left(t_{4}\right)\right)<0$ for $t \geq t_{4}$.

If $\left(b_{2}\right)$ holds, then we obtain by Lemma 2.1

$$
\lim _{t \rightarrow \infty} S_{n-3}(t, x(t))=\lim _{t \rightarrow \infty} S_{n-4}(t, x(t))=\cdots=\lim _{t \rightarrow \infty} x(t)=-\infty
$$

which contradicts with $x(t)>0$ eventually. Hence $\left(\mathrm{b}_{2}\right)$ is impossible.

From $\left(\mathrm{a}_{2}\right)$, we see that $S_{n-3}(t, x(t))$ is strictly increasing on $\left[t_{1}, \infty\right)$ T and exactly one of the following is true:

(a) $S_{n-3}(t, x(t))<0$ for $t \geq t_{1}$;

$\left(\mathrm{b}_{3}\right)$ There exists a $t_{5} \geq t_{1}$ such that $S_{n-3}(t, x(t)) \geq S_{n-3}\left(t_{5}, x\left(t_{5}\right)\right)>0$ for $t \geq t_{5}$.

Therefore we can repeat the above argument and show that the conclusions of Lemma 2.2 hold. The proof is completed.

Remark 2.3 Let $r_{n}(t)=\cdots=r_{1}(t)=1$ and $\mathbf{T}$ be the set of integers. Then Lemma 2.1 and Lemma 2.2 are Lemma 1.8 .10 and Theorem 1.8 .11 of [3] respectively.

\section{Lemma 2.4 Assume that}

$$
\int_{t_{0}}^{\infty} \frac{1}{r_{n-1}(s)}\left\{\int_{s}^{\infty}\left[\frac{1}{r_{n}(u)} \int_{u}^{\infty} q(v) \Delta v\right]^{\frac{1}{\gamma}} \Delta u\right\} \Delta s=\infty
$$

holds and $x(t)$ is an eventually positive solution of Eq. (1.3). Then there exists $T \in\left[t_{0}, \infty\right)$ T sufficiently large such that either of the following cases holds:

(1) $S_{i}(t, x(t))>0$ for any $t \geq T$ and $0 \leq i \leq n$.

(2) $\lim _{t \rightarrow \infty} x(t)=0$.

Proof Since $x(t)$ is an eventually positive solution of Eq. (1.3), there exists a $t_{1} \geq t_{0}$ such that $x(t)>0$ and $x(\tau(t))>0$ on $\left[t_{1}, \infty\right)_{\mathrm{T}}$. It follows from (1.3) that

$$
S_{n}^{\triangle}(t, x(t))=-F(t, x(\tau(t))) \leq-q(t) x^{\gamma}(\tau(t))<0 \quad \text { for } t \geq t_{1} .
$$


By Lemma 2.2, we see that there exists an integer $0 \leq \ell \leq n$, with $\ell+n$ being even, such that $(-1)^{\ell+i} S_{i}(t, x(t))>0$ for $t \geq t_{1}$ and $\ell \leq i \leq n$, and $x(t)$ is eventually monotone.

We claim that $\lim _{t \rightarrow \infty} x(t) \neq 0$ implies $\ell=n$. If not, then $S_{n-1}(t, x(t))<0\left(t \geq t_{1}\right)$ and $S_{n-2}(t, x(t))>0\left(t \geq t_{1}\right)$. It is easy to see that there exist a $t_{2} \geq t_{1}$ and a constant $M>0$ such that $x(\tau(t)) \geq M$ on $\left[t_{2}, \infty\right)_{\mathrm{T}}$. Integrating Eq. (1.3) from $t$ to $\infty$, we get that for $t \geq t_{2}$,

$$
-r_{n}(t)\left[S_{n-1}^{\Delta}(t, x(t))\right]^{\gamma}=-S_{n}(t, x(t)) \leq-M^{\gamma} \int_{t}^{\infty} q(s) \triangle s .
$$

Thus

$$
S_{n-1}(t, x(t)) \leq-M \int_{t}^{\infty}\left[\frac{1}{r_{n}(s)} \int_{s}^{\infty} q(u) \Delta u\right]^{\frac{1}{\gamma}} \triangle s \quad \text { for } t \geq t_{2}
$$

Again, integrating the above inequality from $t_{2}$ to $t$, we obtain that for $t \geq t_{2}$,

$$
S_{n-2}(t, x(t)) \leq S_{n-2}\left(t_{2}, x\left(t_{2}\right)\right)-M \int_{t_{2}}^{t} \frac{1}{r_{n-1}(s)}\left\{\int_{s}^{\infty}\left[\frac{1}{r_{n}(u)} \int_{u}^{\infty} q(v) \Delta v\right]^{\frac{1}{\gamma}} \Delta u\right\} \triangle s .
$$

It follows from (2.1) that $\lim _{t \rightarrow \infty} S_{n-2}(t, x(t))=-\infty$, which is a contradiction to $S_{n-2}(t$, $x(t))>0\left(t \geq t_{1}\right)$. Thus $\ell=n$. The proof is completed.

Lemma 2.5 Let $x(t)$ be a solution of Eq. (1.3) such that case (1) of Lemma 2.4 holds for $t \in[T, \infty)_{\mathrm{T}}$ with some $T \in\left[t_{0}, \infty\right)_{\mathrm{T}}$. Then we have that for $t \in[T, \infty)_{\mathrm{T}}$,

$$
S_{i}(t, x(t)) \geq S_{n}^{\frac{1}{\gamma}}(t, x(t)) \vartheta_{i+1}(t, T), \quad 0 \leq i \leq n-1
$$

and

$$
\frac{S_{n-1}^{\triangle}(t, x(t))}{x^{\sigma}(t)} \geq\left[\frac{\int_{t}^{\infty} q(s) \triangle s}{r_{n}(t)}\right]^{\frac{1}{\gamma}}
$$

where

$$
\vartheta_{i}(t, T)= \begin{cases}\int_{T}^{t}\left[\frac{1}{r_{n}(s)}\right] \frac{1}{\gamma} \Delta s & \text { if } i=n \\ \int_{T}^{t} \frac{\vartheta_{i+1}(s, T)}{r_{i}(s)} \triangle s & \text { if } 1 \leq i \leq n-1 .\end{cases}
$$

Proof Because $x(t)$ is an eventually positive solution of Eq. (1.3), there exists a $T \geq t_{0}$ sufficiently large such that $x(t)>0$ and $x(\tau(t))>0$ for $t \geq T$. Note $S_{n}^{\triangle}(t, x(t))=-F(t, x(\tau(t))) \leq$ $-q(t) x^{\gamma}(\tau(t))<0$, we know that $S_{n}(t, x(t))$ is strictly decreasing on $[T, \infty){ }_{\mathrm{T}}$. Then for $t \geq T$,

$$
\begin{aligned}
S_{n-1}(t, x(t)) & \geq S_{n-1}(t, x(t))-S_{n-1}(T, x(T)) \\
& =\int_{T}^{t}\left[\frac{S_{n}(s, x(s))}{r_{n}(s)}\right]^{\frac{1}{\gamma}} \triangle s \\
& \geq S_{n}^{\frac{1}{\gamma}}(t, x(t)) \vartheta_{n}(t, T),
\end{aligned}
$$




$$
\begin{aligned}
S_{n-2}(t, x(t)) & \geq S_{n-2}(t, x(t))-S_{n-2}(T, x(T)) \\
& =\int_{T}^{t} \frac{S_{n-1}(s, x(s))}{r_{n-1}(s)} \triangle s \\
& \geq S_{n}^{\frac{1}{\gamma}}(t, x(t)) \vartheta_{n-1}(t, T) .
\end{aligned}
$$

Repeating the above process, we have

$$
\begin{aligned}
S_{1}(t, x(t)) & \geq S_{1}(t, x(t))-S_{1}(T, x(T)) \\
& =\int_{T}^{t} \frac{S_{2}(s, x(s))}{r_{2}(s)} \triangle s \\
& \geq S_{n}^{\frac{1}{\gamma}}(t, x(t)) \vartheta_{2}(t, T), \\
S_{0}(t, x(t)) & \geq x(t)-x(T) \\
& =\int_{T}^{t} \frac{S_{1}(s, x(s))}{r_{1}(s)} \triangle s \\
& \geq S_{n}^{\frac{1}{\gamma}}(t, x(t)) \vartheta_{1}(t, T) .
\end{aligned}
$$

Thus it follows

$$
\begin{aligned}
r_{n}(t)\left[S_{n-1}^{\Delta}(t, x(t))\right]^{\gamma} & =S_{n}(t, x(t)) \geq \int_{t}^{\infty} F(s, x(\tau(s))) \triangle s \\
& \geq \int_{t}^{\infty} q(s) x^{\gamma}(\tau(s)) \triangle s \\
& \geq x^{\gamma}(\tau(t)) \int_{t}^{\infty} q(s) \triangle s \\
& \geq x^{\gamma}(\sigma(t)) \int_{t}^{\infty} q(s) \triangle s .
\end{aligned}
$$

That is,

$$
\frac{S_{n-1}^{\Delta}(t, x(t))}{x^{\sigma}(t)} \geq\left[\frac{\int_{t}^{\infty} q(s) \triangle s}{r_{n}(t)}\right]^{\frac{1}{\gamma}} .
$$

The proof is completed.

Lemma 2.6 [25] Let $f: \mathbf{R} \rightarrow \mathbf{R}$ be continuously differentiable and suppose that $g: \mathbf{T} \rightarrow \mathbf{R}$ is delta differentiable. Then $f \circ g$ is delta differentiable and the formula

$$
(f \circ g)^{\triangle}(t)=g^{\triangle}(t) \int_{0}^{1} f^{\prime}\left(h g^{\sigma}(t)+(1-h) g(t)\right) d h
$$

holds.

Lemma 2.7 [23] Suppose that $a$ and $b$ are nonnegative real numbers and $\lambda \geq 1$. Then

$$
\lambda a b^{\lambda-1}-a^{\lambda} \leq(\lambda-1) b^{\lambda},
$$

where the equality holds if and only if $a=b$. 


\section{Main results}

For convenience, we write $\mathbf{D} \equiv\left\{(t, s) \mid t \geq s \geq t_{0}\right\}$. Now we state and prove our main results.

Theorem 3.1 Assume that (2.1) holds. Furthermore, suppose that there exist $G, g \in$ $C_{\text {rd }}(\mathbf{D}, \mathbf{R})$ with $G^{\triangle_{s}} \in C_{\text {rd }}(\mathbf{D}, \mathbf{R})$ such that

$$
G(t, t)=0 \text { for any } t \geq t_{0} \text { and } G(t, s)>0 \text { for any } t>s \geq t_{0} \text {, }
$$

where $G^{\Delta_{s}}$ is the $\triangle$-partial derivative with respect to the second variable, and there exist $m: \mathbf{T} \rightarrow \mathbf{R}$, such that $r_{n}(t) m(t)$ is a delta differentiable function, and a delta differentiable function $M: \mathbf{T} \rightarrow(0, \infty)$ such that

$$
G^{\Delta_{s}}(t, s)+G(t, s) \frac{\beta(s, T)}{M^{\sigma}(s)}=-\frac{g(t, s)}{M^{\sigma}(s)} \sqrt{M(s) G(t, s)} \quad \text { for } t>s \geq t_{0}
$$

and

$$
\limsup _{t \rightarrow \infty} \frac{1}{G(t, T)} \int_{T}^{t}\left[G(t, s) \psi(s, T)-\frac{g_{-}^{2}(t, s) r_{1}(s)}{4 \gamma \eta(s, T)}\right] \triangle s=\infty
$$

for all sufficiently large $T$, where

$$
\begin{aligned}
& \eta(t, T)= \begin{cases}\vartheta_{2}(t, T)\left(\int_{t}^{\infty} q(s) \triangle s\right)^{\frac{1-\gamma}{\gamma}} & \text { if } 0<\gamma \leq 1, \\
\vartheta_{1}^{\gamma-1}(t, T) \vartheta_{2}(t, T) & \text { if } \gamma \geq 1,\end{cases} \\
& \psi(t, T)=M(t) q(t)-M(t)\left(r_{n}(t) m(t)\right)^{\triangle}+\gamma \frac{M(t)}{r_{1}(t)} \eta(t, T)\left(\left(r_{n}(t) m(t)\right)^{\sigma}\right)^{2}, \\
& \beta(t, T)=M^{\triangle}(t)+2 \gamma \frac{M(t) \eta(t, T)}{r_{1}(t)}\left(r_{n}(t) m(t)\right)^{\sigma}
\end{aligned}
$$

and

$$
g_{-}(t, s)=\max \{0,-g(t, s)\} .
$$

Then every solution $x(t)$ of Eq. (1.3) is either oscillatory or tends to zero.

Proof Assume that Eq. (1.3) has a nonoscillatory solution $x(t)$ on $\left[t_{0}, \infty\right)_{\mathrm{T}}$. Then, without loss of generality, there is a $T \geq t_{0}$, sufficiently large, such that $x(t)>0$ for $t \geq T$. By Lemma 2.4, there are two possible cases:

(1) $S_{i}(t, x(t))>0$ for any $t \geq T$ and $0 \leq i \leq n$.

(2) $\lim _{t \rightarrow \infty} x(t)=0$.

If case (1) holds, then set

$$
\begin{aligned}
\omega(t) & =M(t)\left[\frac{S_{n}(t, x(t))}{x^{\gamma}(t)}+r_{n}(t) m(t)\right] \\
& =M(t) r_{n}(t)\left[\left(\frac{S_{n-1}^{\triangle}(t, x(t))}{x(t)}\right)^{\gamma}+m(t)\right],
\end{aligned}
$$


we have

$$
\begin{aligned}
\omega^{\Delta}(t)= & \left(M(t) \frac{S_{n}(t, x(t))}{x^{\gamma}(t)}\right)^{\Delta}+\left(M(t) r_{n}(t) m(t)\right)^{\Delta} \\
= & \frac{M(t)}{x^{\gamma}(t)} S_{n}^{\Delta}(t, x(t))+\left(\frac{M(t)}{x^{\gamma}(t)}\right)^{\Delta} S_{n}^{\sigma}(t, x(t)) \\
& +M(t)\left(r_{n}(t) m(t)\right)^{\Delta}+M^{\triangle}(t)\left(r_{n}(t) m(t)\right)^{\sigma} \\
= & \frac{M(t)}{x^{\gamma}(t)} S_{n}^{\Delta}(t, x(t))+\left(\frac{M^{\Delta}(t)}{x^{\gamma \sigma}(t)}-\frac{M(t)\left(x^{\gamma}(t)\right)^{\Delta}}{x^{\gamma}(t) x^{\gamma \sigma}(t)}\right) S_{n}^{\sigma}(t, x(t)) \\
& +M(t)\left(r_{n}(t) m(t)\right)^{\Delta}+M^{\Delta}(t)\left(r_{n}(t) m(t)\right)^{\sigma} .
\end{aligned}
$$

It follows from (1.3) and the definition of $\omega(t)$ that for all $t \geq T$,

$$
\begin{aligned}
\omega^{\Delta}(t)= & -\frac{M(t)}{x^{\gamma}(t)} F(t, x(\tau(t)))+M(t)\left(r_{n}(t) m(t)\right)^{\Delta} \\
& +\frac{M^{\triangle}(t)}{M^{\sigma}(t)} \omega^{\sigma}(t)-M(t) \frac{\left(x^{\gamma}(t)\right)^{\triangle}}{x^{\gamma}(t)} \frac{S_{n}^{\sigma}(t, x(t))}{x^{\gamma \sigma}(t)} .
\end{aligned}
$$

Using the fact that $F(t, x(\tau(t))) \geq q(t) x^{\gamma}(\tau(t))$ and $x(t)$ is increasing on $[T, \infty)$, we get

$$
\begin{aligned}
\omega^{\triangle}(t) \leq & -M(t) q(t)+M(t)\left(r_{n}(t) m(t)\right)^{\Delta} \\
& +\frac{M^{\Delta}(t)}{M^{\sigma}(t)} \omega^{\sigma}(t)-M(t) \frac{\left(x^{\gamma}(t)\right)^{\Delta}}{x^{\gamma}(t)} \frac{S_{n}^{\sigma}(t, x(t))}{x^{\gamma \sigma}(t)} .
\end{aligned}
$$

Now we consider the following two cases.

Case 1: If $0<\gamma \leq 1$, then it follows from $x^{\Delta}(t)>0$ and Lemma 2.6 that $x^{\sigma}(t) \geq x(t)$ and

$$
\begin{aligned}
\left(x^{\gamma}(t)\right)^{\Delta} & =\gamma x^{\Delta}(t) \int_{0}^{1}\left(h x^{\sigma}(t)+(1-h) x(t)\right)^{\gamma-1} d h \\
& \geq \gamma x^{\Delta}(t) \int_{0}^{1}\left(h x^{\sigma}(t)+(1-h) x^{\sigma}(t)\right)^{\gamma-1} d h \\
& =\gamma\left(x^{\sigma}(t)\right)^{\gamma-1} x^{\Delta}(t) .
\end{aligned}
$$

By (3.9) and (3.10), we have

$$
\begin{aligned}
\omega^{\Delta}(t) \leq & -M(t) q(t)+M(t)\left(r_{n}(t) m(t)\right)^{\Delta} \\
& +\frac{M^{\Delta}(t)}{M^{\sigma}(t)} \omega^{\sigma}(t)-\gamma M(t) \frac{x^{\Delta}(t)}{x^{\sigma}(t)} \frac{x^{\gamma \sigma}(t)}{x^{\gamma}(t)} \frac{S_{n}^{\sigma}(t, x(t))}{x^{\gamma \sigma}(t)} .
\end{aligned}
$$

It follows from Lemma 2.5 that

$$
\begin{aligned}
& \frac{x^{\Delta}(t)}{S_{n}^{\frac{1}{\gamma}}(t, x(t))} \geq \frac{\vartheta_{2}(t, T)}{r_{1}(t)}, \quad \frac{x(t)}{S_{n}^{\frac{1}{\gamma}}(t, x(t))} \geq \vartheta_{1}(t, T), \\
& \frac{S_{n-1}^{\Delta}(t, x(t))}{x^{\sigma}(t)} \geq\left[\frac{\int_{t}^{\infty} q(s) \Delta s}{r_{n}(t)}\right]^{\frac{1}{\gamma}} .
\end{aligned}
$$


Then

$$
\begin{aligned}
\frac{x^{\triangle}(t)}{x^{\sigma}(t)} & =r_{n}^{\frac{1}{\gamma}-1}(t) \frac{S_{n}(t, x(t))}{x^{\gamma \sigma}(t)}\left(\frac{S_{n-1}^{\Delta}(t, x(t))}{x^{\sigma}(t)}\right)^{1-\gamma} \frac{x^{\triangle}(t)}{S_{n}^{\frac{1}{\gamma}}(t, x(t))} \\
& \geq r_{n}^{\frac{1}{\gamma}-1}(t) \frac{S_{n}(t, x(t))}{x^{\gamma \sigma}(t)}\left(\left(\frac{\int_{t}^{\infty} q(s) \triangle s}{r_{n}(t)}\right)^{\frac{1}{\gamma}}\right)^{1-\gamma} \frac{\vartheta_{2}(t, T)}{r_{1}(t)} \\
& \geq \frac{\vartheta_{2}(t, T)}{r_{1}(t)}\left(\int_{t}^{\infty} q(s) \triangle s\right)^{\frac{1-\gamma}{\gamma}} \frac{S_{n}^{\sigma}(t, x(t))}{x^{\gamma \sigma}(t)} .
\end{aligned}
$$

Combining (3.11) with (3.13), we get

$$
\begin{aligned}
\omega^{\triangle}(t) \leq & -M(t) q(t)+M(t)\left(r_{n}(t) m(t)\right)^{\triangle} \\
& +\frac{M^{\triangle}(t)}{M^{\sigma}(t)} \omega^{\sigma}(t)-\gamma M(t) \frac{\vartheta_{2}(t, T)}{r_{1}(t)}\left[\int_{t}^{\infty} q(s) \triangle s\right]^{\frac{1-\gamma}{\gamma}}\left(\frac{S_{n}^{\sigma}(t, x(t))}{x^{\gamma \sigma}(t)}\right)^{2} .
\end{aligned}
$$

Case 2: If $\gamma \geq 1$, then it follows from $x^{\Delta}(t)>0$ and Lemma 2.6 that $x^{\sigma}(t) \geq x(t)$ and

$$
\begin{aligned}
\left(x^{\gamma}(t)\right)^{\triangle} & =\gamma x^{\triangle}(t) \int_{0}^{1}\left(h x^{\sigma}(t)+(1-h) x(t)\right)^{\gamma-1} d h \\
& \geq \gamma x^{\triangle}(t) \int_{0}^{1}(h x(t)+(1-h) x(t))^{\gamma-1} d h \\
& =\gamma(x(t))^{\gamma-1} x^{\Delta}(t) .
\end{aligned}
$$

By (3.9) and (3.15), we have

$$
\begin{aligned}
\omega^{\triangle}(t) \leq & -M(t) q(t)+M(t)\left(r_{n}(t) m(t)\right)^{\Delta} \\
& +\frac{M^{\Delta}(t)}{M^{\sigma}(t)} \omega^{\sigma}(t)-\gamma M(t) \frac{x^{\Delta}(t)}{x(t)} \frac{S_{n}^{\sigma}(t, x(t))}{x^{\gamma \sigma}(t)} .
\end{aligned}
$$

It follows from (3.12) that

$$
\begin{aligned}
\frac{x^{\Delta}(t)}{x(t)} & =\frac{S_{n}(t, x(t))}{x^{\gamma}(t)}\left(\frac{x(t)}{S_{n}^{\frac{1}{\gamma}}(t, x(t))}\right)^{\gamma-1} \frac{x^{\Delta}(t)}{S_{n}^{\frac{1}{\gamma}}(t, x(t))} \\
& \geq \frac{S_{n}(t, x(t))}{x^{\gamma}(t)}\left(\vartheta_{1}(t, T)\right)^{\gamma-1} \frac{\vartheta_{2}(t, T)}{r_{1}(t)} \\
& \geq\left(\vartheta_{1}(t, T)\right)^{\gamma-1} \frac{\vartheta_{2}(t, T)}{r_{1}(t)} \frac{S_{n}^{\sigma}(t, x(t))}{x^{\gamma \sigma}(t)} .
\end{aligned}
$$

Combining (3.16) with (3.17) gives

$$
\begin{aligned}
\omega^{\triangle}(t) \leq & -M(t) q(t)+M(t)\left(r_{n}(t) m(t)\right)^{\triangle} \\
& +\frac{M^{\triangle}(t)}{M^{\sigma}(t)} \omega^{\sigma}(t)-\gamma M(t)\left(\vartheta_{1}(t, T)\right)^{\gamma-1} \frac{\vartheta_{2}(t, T)}{r_{1}(t)}\left(\frac{S_{n}^{\sigma}(t, x(t))}{x^{\gamma \sigma}(t)}\right)^{2} .
\end{aligned}
$$


Noting that the definitions of $\eta(t, T), \psi(t, T)$ and $\beta(t, T)$. It follows from (3.14), (3.18) and the fact

$$
\frac{S_{n}^{\sigma}(t, x(t))}{x^{\gamma \sigma}(t)}=\frac{\omega^{\sigma}(t)}{M^{\sigma}(t)}-r_{n}^{\sigma}(t) m^{\sigma}(t)
$$

that for $\gamma>0$,

$$
\psi(t, T) \leq-\omega^{\Delta}(t)+\frac{\beta(t, T)}{M^{\sigma}(t)} \omega^{\sigma}(t)-\frac{\gamma M(t) \eta(t, T)}{r_{1}(t)\left(M^{\sigma}(t)\right)^{2}}\left(\omega^{\sigma}(t)\right)^{2} .
$$

Multiplying both sides of (3.19), with $t$ replaced by $s$, by $G(t, s)$ and integrating with respect to $s$ from $T$ to $t(t \geq T)$, one gets

$$
\begin{aligned}
\int_{T}^{t} G(t, s) \psi(s, T) \triangle s \leq & -\int_{T}^{t} G(t, s) \omega^{\Delta}(s) \triangle s+\int_{T}^{t} \frac{G(t, s) \beta(s, T)}{M^{\sigma}(s)} \omega^{\sigma}(s) \triangle s \\
& -\int_{T}^{t} \frac{\gamma G(t, s) M(s) \eta(s, T)}{r_{1}(s)\left(M^{\sigma}(s)\right)^{2}}\left(\omega^{\sigma}(s)\right)^{2} \triangle s .
\end{aligned}
$$

Integrating by parts and using (3.1) and (3.2), we have

$$
\begin{aligned}
\int_{T}^{t} G(t, s) \psi(s, T) \triangle s \leq & G(t, T) \omega(T)+\int_{T}^{t} G^{\Delta_{s}}(t, s) \omega^{\sigma}(s) \triangle s+\int_{T}^{t} \frac{G(t, s) \beta(s, T)}{M^{\sigma}(s)} \omega^{\sigma}(s) \triangle s \\
& -\int_{T}^{t} \frac{\gamma G(t, s) M(s) \eta(s, T)}{r_{1}(s)\left(M^{\sigma}(s)\right)^{2}}\left(\omega^{\sigma}(s)\right)^{2} \triangle s \\
= & G(t, T) \omega(T)+\int_{T}^{t}\left[-\frac{g(t, s)}{M^{\sigma}(s)} \sqrt{M(s) G(t, s)} \omega^{\sigma}(s)\right. \\
& \left.-\frac{\gamma G(t, s) M(s) \eta(s, T)}{r_{1}(s)\left(M^{\sigma}(s)\right)^{2}}\left(\omega^{\sigma}(s)\right)^{2}\right] \triangle s \\
\leq & G(t, T) \omega(T)+\int_{T}^{t}\left[\frac{g_{-}(t, s)}{M^{\sigma}(s)} \sqrt{M(s) G(t, s)} \omega^{\sigma}(s)\right. \\
& \left.-\frac{\gamma G(t, s) M(s) \eta(s, T)}{r_{1}(s)\left(M^{\sigma}(s)\right)^{2}}\left(\omega^{\sigma}(s)\right)^{2}\right] \triangle s .
\end{aligned}
$$

It is easy to check that

$$
\begin{aligned}
& \frac{g_{-}(t, s)}{M^{\sigma}(s)} \sqrt{M(s) G(t, s)} \omega^{\sigma}(s)-\frac{\gamma G(t, s) M(s) \eta(s, T)}{r_{1}(s)\left(M^{\sigma}(s)\right)^{2}}\left(\omega^{\sigma}(s)\right)^{2} \\
& \quad=\frac{g_{-}^{2}(t, s) r_{1}(s)}{4 \gamma \eta(s, T)}-\frac{\gamma M(s) \eta(s, T)}{r_{1}(s)\left(M^{\sigma}(s)\right)^{2}}\left(\sqrt{G(t, s)} \omega^{\sigma}(s)-\frac{g_{-}(t, s) M^{\sigma}(s) r_{1}(s)}{2 \gamma \sqrt{M(s)} \eta(s, T)}\right)^{2},
\end{aligned}
$$

which implies

$$
\frac{g_{-}(t, s)}{M^{\sigma}(s)} \sqrt{M(s) G(t, s)} \omega^{\sigma}(s)-\frac{\gamma G(t, s) M(s) \eta(s, T)}{r_{1}(s)\left(M^{\sigma}(s)\right)^{2}}\left(\omega^{\sigma}(s)\right)^{2} \leq \frac{g_{-}^{2}(t, s) r_{1}(s)}{4 \gamma \eta(s, T)} .
$$

Combining (3.21) with (3.20), it follows

$$
\frac{1}{G(t, T)} \int_{T}^{t}\left[G(t, s) \psi(s, T)-\frac{g_{-}^{2}(t, s) r_{1}(s)}{4 \gamma \eta(s, T)}\right] \triangle s \leq \omega(T),
$$


which contradicts assumption (3.3). Thus every solution $x(t)$ of Eq. (1.3) is either oscillatory or tends to zero. The proof is completed.

Theorem 3.2 Assume that (2.1) holds. Furthermore, suppose that there exist $H, h \in$ $C_{\mathrm{rd}}(\mathbf{D}, \mathbf{R})$ with $H^{\triangle_{s}} \in C_{\mathrm{rd}}(\mathbf{D}, \mathbf{R})$ such that

$$
H(t, t)=0 \text { for any } t \geq t_{0} \text { and } H(t, s)>0 \text { for any } t>s \geq t_{0} \text {, }
$$

where $H^{\triangle_{s}}$ is the $\triangle$-partial derivative with respect to the second variable, and there exists a delta differentiable function $M: \mathbf{T} \rightarrow(0, \infty)$ such that

$$
H^{\triangle_{s}}(t, s)+H(t, s) \frac{M^{\triangle}(s)}{M^{\sigma}(s)}=-\frac{h(t, s)}{M^{\sigma}(s)}(M(s) H(t, s))^{\frac{\gamma}{\gamma+1}} \quad \text { for } t>s \geq t_{0}
$$

and

$$
\limsup _{t \rightarrow \infty} \frac{1}{H(t, T)} \int_{T}^{t}\left[M(s) q(s) H(t, s)-\frac{h_{-}^{\gamma+1}(t, s) r_{1}^{\gamma}(s)}{(\gamma+1)^{\gamma+1} \vartheta_{2}^{\gamma}(s, T)}\right] \triangle s=\infty
$$

for all sufficiently large $T$, where

$$
h_{-}(t, s)=\max \{0,-h(t, s)\} \text {. }
$$

Then every solution $x(t)$ of Eq. (1.3) is either oscillatory or tends to zero.

Proof Assume that Eq. (1.3) has a nonoscillatory solution $x(t)$ on $\left[t_{0}, \infty\right)_{\mathrm{T}}$. Then, without loss of generality, there is a $T \geq t_{0}$, sufficiently large, such that $x(t)>0$ for $t \geq T$. By Lemma 2.4, there are two possible cases:

(1) $S_{i}(t, x(t))>0$ for any $t \geq T$ and $0 \leq i \leq n$.

(2) $\lim _{t \rightarrow \infty} x(t)=0$.

If case (1) holds, then set

$$
\omega(t)=M(t)\left[\frac{S_{n}(t, x(t))}{x^{\gamma}(t)}\right]=M(t) r_{n}(t)\left[\left(\frac{S_{n-1}^{\triangle}(t, x(t))}{x(t)}\right)^{\gamma}\right] .
$$

By (3.9), we have

$$
\begin{aligned}
\omega^{\triangle}(t) & \leq-M(t) q(t)+\frac{M^{\triangle}(t)}{M^{\sigma}(t)} \omega^{\sigma}(t)-M(t) \frac{\left(x^{\gamma}(t)\right)^{\triangle}}{x^{\gamma}(t)} \frac{S_{n}^{\sigma}(t, x(t))}{x^{\gamma \sigma}(t)} \\
& \leq-M(t) q(t)+\frac{M^{\triangle}(t)}{M^{\sigma}(t)} \omega^{\sigma}(t)-\frac{M(t)}{M^{\sigma}(t)} \frac{\left(x^{\gamma}(t)\right)^{\triangle}}{x^{\gamma}(t)} \omega^{\sigma}(t) .
\end{aligned}
$$

It follows from Lemma 2.6 that

$$
\begin{aligned}
\left(x^{\gamma}(t)\right)^{\Delta} & =\gamma x^{\triangle}(t) \int_{0}^{1}\left(h x^{\sigma}(t)+(1-h) x(t)\right)^{\gamma-1} d h \\
& \geq \begin{cases}\gamma\left(x^{\sigma}(t)\right)^{\gamma-1} x^{\triangle}(t) & \text { if } 0<\gamma \leq 1, \\
\gamma(x(t))^{\gamma-1} x^{\triangle}(t) & \text { if } \gamma \geq 1 .\end{cases}
\end{aligned}
$$


Case 1. If $0<\gamma \leq 1$, then

$$
\omega^{\triangle}(t) \leq-M(t) q(t)+\frac{M^{\Delta}(t)}{M^{\sigma}(t)} \omega^{\sigma}(t)-\gamma \frac{M(t)}{M^{\sigma}(t)} \frac{x^{\Delta}(t)}{x^{\sigma}(t)} \frac{x^{\gamma \sigma}(t)}{x^{\gamma}(t)} \omega^{\sigma}(t) .
$$

Case 2. If $\gamma \geq 1$, then

$$
\omega^{\triangle}(t) \leq-M(t) q(t)+\frac{M^{\Delta}(t)}{M^{\sigma}(t)} \omega^{\sigma}(t)-\gamma \frac{M(t)}{M^{\sigma}(t)} \frac{x^{\Delta}(t)}{x(t)} \omega^{\sigma}(t) .
$$

Noting that $x^{\sigma}(t) \geq x(t)$, we have

$$
\omega^{\triangle}(t) \leq-M(t) q(t)+\frac{M^{\triangle}(t)}{M^{\sigma}(t)} \omega^{\sigma}(t)-\gamma \frac{M(t)}{M^{\sigma}(t)} \frac{x^{\triangle}(t)}{x^{\sigma}(t)} \omega^{\sigma}(t) .
$$

By (3.12), we obtain

$$
\begin{aligned}
\omega^{\triangle}(t) & \leq-M(t) q(t)+\frac{M^{\triangle}(t)}{M^{\sigma}(t)} \omega^{\sigma}(t)-\gamma \frac{M(t)}{M^{\sigma}(t)} \frac{\vartheta_{2}(t, T)}{r_{1}(t)} \frac{S_{n}^{\frac{1}{\gamma}}(t, x(t))}{x^{\sigma}(t)} \omega^{\sigma}(t) \\
& \leq-M(t) q(t)+\frac{M^{\triangle}(t)}{M^{\sigma}(t)} \omega^{\sigma}(t)-\gamma \frac{M(t)}{\left(M^{\sigma}(t)\right)^{\lambda}} \frac{\vartheta_{2}(t, T)}{r_{1}(t)}\left(\omega^{\sigma}(t)\right)^{\lambda},
\end{aligned}
$$

where $\lambda=1+\frac{1}{\gamma}$. Multiplying both sides of (3.32), with $t$ replaced by $s$, by $H(t, s)$ and integrating with respect to $s$ from $T$ to $t(t \geq T)$, one gets

$$
\begin{aligned}
\int_{T}^{t} H(t, s) M(s) q(s) \triangle s \leq & -\int_{T}^{t} H(t, s) \omega^{\triangle}(s) \triangle s+\int_{T}^{t} H(t, s) \frac{M^{\Delta}(s)}{M^{\sigma}(s)} \omega^{\sigma}(s) \triangle s \\
& -\int_{T}^{t} \gamma H(t, s) \frac{M(s) \vartheta_{2}(s, T)}{\left(M^{\sigma}(s)\right)^{\lambda} r_{1}(s)}\left(\omega^{\sigma}(s)\right)^{\lambda} \triangle s .
\end{aligned}
$$

Integrating by parts and using (3.22) and (3.23), we have

$$
\begin{aligned}
\int_{T}^{t} H(t, s) M(s) q(s) \triangle s \leq & H(t, T) \omega(T)+\int_{T}^{t} H^{\triangle_{s}}(t, s) \omega^{\sigma}(s) \triangle s \\
& +\int_{T}^{t} H(t, s) \frac{M^{\Delta}(s)}{M^{\sigma}(s)} \omega^{\sigma}(s) \triangle s \\
& -\int_{T}^{t} \gamma H(t, s) \frac{M(s) \vartheta_{2}(s, T)}{\left(M^{\sigma}(s)\right)^{\lambda} r_{1}(s)}\left(\omega^{\sigma}(s)\right)^{\lambda} \triangle s \\
\leq & H(t, T) \omega(T)+\int_{T}^{t}\left[-\frac{h(t, s)}{M^{\sigma}(s)}(M(s) H(t, s))^{\frac{\gamma}{\gamma+1}} \omega^{\sigma}(s)\right. \\
& \left.-\gamma H(t, s) \frac{M(s) \vartheta_{2}(s, T)}{\left(M^{\sigma}(s)\right)^{\lambda} r_{1}(s)}\left(\omega^{\sigma}(s)\right)^{\lambda}\right] \triangle s \\
\leq & H(t, T) \omega(T)+\int_{T}^{t}\left[\frac{h_{-}(t, s)}{M^{\sigma}(s)}(M(s) H(t, s))^{\frac{1}{\lambda}} \omega^{\sigma}(s)\right. \\
& \left.-\gamma H(t, s) \frac{M(s) \vartheta_{2}(s, T)}{\left(M^{\sigma}(s)\right)^{\lambda} r_{1}(s)}\left(\omega^{\sigma}(s)\right)^{\lambda}\right] \triangle s .
\end{aligned}
$$


Write

$$
A^{\lambda}=\gamma \frac{H(t, s) M(s) \vartheta_{2}(s, T)}{r_{1}(s)\left(M^{\sigma}(s)\right)^{\lambda}}\left(\omega^{\sigma}(s)\right)^{\lambda}, \quad B^{\lambda-1}=\frac{h_{-}(t, s) r_{1}^{\frac{1}{\lambda}}(s)}{\lambda \gamma^{\frac{1}{\lambda}} \vartheta_{2}^{\frac{1}{\lambda}}(s, T)} .
$$

It follows from Lemma 2.7 that

$$
\frac{h_{-}(t, s)}{M^{\sigma}(s)}(M(s) H(t, s))^{\frac{1}{\lambda}} \omega^{\sigma}(s)-\gamma H(t, s) \frac{M(s) \vartheta_{2}(s, T)}{\left(M^{\sigma}(s)\right)^{\lambda} r_{1}(s)}\left(\omega^{\sigma}(s)\right)^{\lambda} \leq \frac{h_{-}^{\gamma+1}(t, s) r_{1}^{\gamma}(s)}{(\gamma+1)^{\gamma+1} \vartheta_{2}^{\gamma}(s, T)} .
$$

Combining the above inequality with (3.33) gives

$$
\int_{T}^{t}\left[M(s) q(s) H(t, s)-\frac{h_{-}^{\gamma+1}(t, s) r_{1}^{\gamma}(s)}{(\gamma+1)^{\gamma+1} \vartheta_{2}^{\gamma}(s, T)}\right] \triangle s \leq H(t, T) \omega(T)
$$

which implies

$$
\frac{1}{H(t, T)} \int_{T}^{t}\left[M(s) q(s) H(t, s)-\frac{h_{-}^{\gamma+1}(t, s) r_{1}^{\gamma}(s)}{(\gamma+1)^{\gamma+1} \vartheta_{2}^{\gamma}(s, T)}\right] \triangle s \leq \omega(T),
$$

which contradicts assumption (3.24). Thus every solution $x(t)$ of Eq. (1.3) is either oscillatory or tends to zero. The proof is completed.

Theorem 3.3 Assume that (2.1) holds. Furthermore, suppose that for all sufficiently large $T$,

$$
\limsup _{t \rightarrow \infty} \vartheta_{1}^{\gamma}(t, T) \int_{t}^{\infty} q(s) \Delta s>1
$$

holds. Then every solution $x(t)$ of Eq. (1.3) is either oscillatory or tends to zero.

Proof Assume that Eq. (1.3) has a nonoscillatory solution $x(t)$ on $\left[t_{0}, \infty\right)_{\mathbf{T}}$. Then, without loss of generality, there is a $T \geq t_{0}$, sufficiently large, such that $x(t)>0$ for $t \geq T$. By Lemma 2.4, there are two possible cases:

(1) $S_{i}(t, x(t))>0$ for any $t \geq T$ and $0 \leq i \leq n$.

(2) $\lim _{t \rightarrow \infty} x(t)=0$.

If case (1) holds, then using the fact that $S_{n}^{\triangle}(t, x(t))<0$, we obtain

$$
S_{n}(t, x(t)) \geq \int_{t}^{\infty} F(s, x(\tau(s))) \triangle s \geq x^{\gamma}(t) \int_{t}^{\infty} q(s) \triangle s,
$$

which implies

$$
\int_{t}^{\infty} q(s) \triangle s \leq\left(\frac{S_{n}^{\frac{1}{\gamma}}(t, x(t))}{x(t)}\right)^{\gamma}
$$

Combining (3.35) with (3.12) gives

$$
\vartheta_{1}^{\gamma}(t, T) \int_{t}^{\infty} q(s) \triangle s \leq 1
$$


Therefore

$$
\limsup _{t \rightarrow \infty} \vartheta_{1}^{\gamma}(t, T) \int_{t}^{\infty} q(s) \triangle s \leq 1
$$

which contradicts assumption (3.34). Thus every solution $x(t)$ of Eq. (1.3) is either oscillatory or tends to zero. The proof is completed.

\section{Examples}

In this section, we give some examples to illustrate our main results.

Example 4.1 Consider the following higher-order dynamic equation:

$$
S_{n}^{\triangle}(t, x(t))+\frac{\rho}{t^{\frac{4}{3}}} x^{3}(\tau(t))=0, \quad t \in 2^{\mathrm{Z}}, t \geq 2,
$$

where $n \geq 2, \gamma=3, \rho$ is a positive constant, $S_{k}(t, x(t))(0 \leq k \leq n)$ are as in Eq. (1.3) with $r_{n}(t)=t^{3}, r_{n-1}(t)=\cdots=r_{1}(t)=1$ and $\tau$ is defined as in $\left(\mathrm{H}_{4}\right)$. If $\rho>\frac{1}{12}$, then every solution of Eq. (4.1) is either oscillatory or tends to zero.

Proof Note that

$$
\begin{aligned}
& \int_{t_{0}}^{t}\left[\frac{1}{r_{n}(s)}\right]^{\frac{1}{\gamma}} \Delta s=\int_{2}^{t} \frac{1}{s} \Delta s=\log _{2} t-1 \rightarrow \infty \quad(t \rightarrow \infty), \\
& \int_{t_{0}}^{\infty} \frac{\Delta s}{r_{i}(s)}=\int_{2}^{\infty} \Delta s=\infty \quad \text { for } 1 \leq i \leq n-1
\end{aligned}
$$

and

$$
\begin{aligned}
\int_{t_{0}}^{\infty} & \frac{1}{r_{n-1}(s)}\left\{\int_{s}^{\infty}\left[\frac{1}{r_{n}(u)} \int_{u}^{\infty} q(v) \Delta v\right]^{\frac{1}{\gamma}} \Delta u\right\} \triangle s \\
= & \int_{2}^{\infty}\left\{\int_{s}^{\infty}\left[\frac{1}{u^{3}} \int_{u}^{\infty} \frac{\rho}{v^{\frac{4}{3}}} \Delta v\right]^{\frac{1}{3}} \Delta u\right\} \triangle s \\
& =\left(\frac{\rho}{1-2^{-\frac{1}{3}}}\right)^{\frac{1}{3}} \int_{2}^{\infty}\left\{\int_{s}^{\infty} \frac{1}{u^{\frac{10}{9}}} \Delta u\right\} \triangle s \\
& =\left(\frac{\rho}{1-2^{-\frac{1}{3}}}\right)^{\frac{1}{3}} \frac{1}{1-2^{-\frac{1}{9}}} \int_{2}^{\infty} s^{-\frac{1}{9}} \triangle s \\
& \left.=\left(\frac{\rho}{1-2^{-\frac{1}{3}}}\right)^{\frac{1}{3}} \frac{1}{1-2^{-\frac{1}{9}}} \frac{1}{2^{\frac{8}{9}}-1^{t \rightarrow \infty}} \lim _{t \rightarrow t^{\frac{8}{9}}}-2^{\frac{8}{9}}\right) \\
& =\infty
\end{aligned}
$$

Take $M(t)=t, m(t)=\frac{1}{t^{4}}$ and $G(t, s)=1$ if $t>s \geq 2$ and $G(t, t)=0$ if $t \geq 2$, then

$$
\begin{aligned}
& \psi(s, T)=\frac{\rho}{s^{\frac{1}{3}}}+\frac{1}{\sigma(s)}+\frac{3 s \eta(s, T)}{\sigma^{2}(s)}, \\
& \beta(s, T)=1+\frac{6 s \eta(s, T)}{\sigma(s)}
\end{aligned}
$$


and

$$
g(t, s)=-\frac{1}{\sqrt{s}}\left(1+\frac{6 s \eta(s, T)}{\sigma(s)}\right) .
$$

Note that $\vartheta_{n}(t, T)=\int_{T}^{t}\left[\frac{1}{r_{n}(s)}\right]^{\frac{1}{\gamma}} \triangle s=\int_{T}^{t} \frac{1}{s} \triangle s=\log _{2} t-\log _{2} T$. It is easy to see that

$$
\lim _{t \rightarrow \infty} \vartheta_{2}(t, T)=\lim _{t \rightarrow \infty} \vartheta_{1}(t, T)=\lim _{t \rightarrow \infty} \vartheta_{n}(t, T)=\infty
$$

From (3.4) and (4.2), we can find $T^{*}$ such that $\eta(t, T) \geq 1$ for all $t \geq T^{*}$. Therefore we have that if $\rho>\frac{1}{12}$, then

$$
\begin{aligned}
& \limsup _{t \rightarrow \infty} \frac{1}{G(t, T)} \int_{T}^{t}\left[G(t, s) \psi(s, T)-\frac{g_{-}^{2}(t, s) r_{1}(s)}{4 \gamma \eta(s, T)}\right] \triangle s \\
& \quad=\limsup _{t \rightarrow \infty} \int_{T}^{t}\left[\frac{\rho}{s^{\frac{1}{3}}}-\frac{1}{12 s \eta(s, T)}\right] \triangle s \\
& \quad \geq\left(\rho-\frac{1}{12}\right) \limsup _{t \rightarrow \infty} \int_{T^{*}}^{t} \frac{1}{s^{\frac{1}{3}}} \triangle s \\
& \quad=\infty .
\end{aligned}
$$

Thus conditions $\left(\mathrm{H}_{3}\right),(2.1)$ and (3.3) are satisfied. By Theorem 3.1, every solution of Eq. (4.1) is either oscillatory or tends to zero if $\rho>\frac{1}{12}$. The proof is completed.

Example 4.2 Consider the following higher-order dynamic equation:

$$
S_{n}^{\triangle}(t, x(t))+\frac{\rho}{t^{\frac{4}{3}}} x^{\frac{2}{3}}(\tau(t))=0, \quad t \in 2^{\mathbf{Z}}, t \geq 2,
$$

where $n \geq 2, \gamma=\frac{2}{3}, S_{k}(t, x(t))(0 \leq k \leq n)$ are as in Eq. (1.3) with $r_{n}(t)=t^{\frac{1}{2}}, r_{n-1}(t)=t^{\frac{1}{4}}$, $r_{n-2}(t)=\cdots=r_{1}(t)=t, \tau$ is defined as in $\left(\mathrm{H}_{4}\right)$ and $\rho$ is a positive constant. If $\rho>\frac{1}{\left(\frac{5}{3}\right)^{\frac{5}{3}}}$, then every solution of Eq. (4.3) is either oscillatory or tends to zero.

Proof Note that

$$
\begin{aligned}
& \int_{t_{0}}^{t}\left[\frac{1}{r_{n}(s)}\right]^{\frac{1}{\gamma}} \Delta s=\int_{2}^{t} \frac{1}{s^{\frac{3}{4}}} \Delta s=\frac{t^{\frac{1}{4}}-2^{\frac{1}{4}}}{2^{\frac{1}{4}}-1} \rightarrow \infty \quad(t \rightarrow \infty), \\
& \int_{t_{0}}^{t} \frac{1}{r_{n-1}(s)} \triangle s=\int_{2}^{t} \frac{1}{s^{\frac{1}{4}}} \Delta s=\frac{t^{\frac{3}{4}}-2^{\frac{3}{4}}}{2^{\frac{3}{4}}-1} \rightarrow \infty \quad(t \rightarrow \infty), \\
& \int_{t_{0}}^{t} \frac{1}{r_{i}(s)} \triangle s=\int_{2}^{t} \frac{1}{s} \Delta s=\log _{2} t-1 \rightarrow \infty \quad(t \rightarrow \infty) \text { for } 1 \leq i \leq n-2
\end{aligned}
$$

and

$$
\begin{gathered}
\int_{t_{0}}^{\infty} \frac{1}{r_{n-1}(s)}\left\{\int_{s}^{\infty}\left[\frac{1}{r_{n}(u)} \int_{u}^{\infty} q(v) \Delta v\right]^{\frac{1}{\gamma}} \Delta u\right\} \Delta s \\
=\int_{2}^{\infty} \frac{1}{s^{\frac{1}{4}}}\left\{\int_{s}^{\infty}\left[\frac{1}{u^{\frac{1}{2}}} \int_{u}^{\infty} \frac{\rho}{v^{\frac{4}{3}}} \Delta v\right]^{\frac{3}{2}} \Delta u\right\} \triangle s
\end{gathered}
$$




$$
\begin{aligned}
& =\left(\frac{\rho}{1-2^{-\frac{1}{3}}}\right)^{\frac{3}{2}} \int_{2}^{\infty} \frac{1}{s^{\frac{1}{4}}}\left\{\int_{s}^{\infty} \frac{1}{u^{\frac{5}{4}}} \Delta u\right\} \Delta s \\
& =\left(\frac{\rho}{1-2^{-\frac{1}{3}}}\right)^{\frac{3}{2}} \frac{1}{1-2^{-\frac{1}{4}}} \int_{2}^{\infty} \frac{1}{s^{\frac{1}{2}}} \Delta s \\
& =\left(\frac{\rho}{1-2^{-\frac{1}{3}}}\right)^{\frac{3}{2}} \frac{1}{1-2^{-\frac{1}{4}}} \lim _{t \rightarrow \infty} \frac{t^{\frac{1}{2}}-2^{\frac{1}{2}}}{2^{\frac{1}{2}}-1} \\
& =\infty
\end{aligned}
$$

Note that

$$
\begin{aligned}
& \vartheta_{2}(t, T)=\int_{T}^{t} \frac{1}{r_{2}\left(u_{n-1}\right)}\left[\int _ { T } ^ { u _ { n - 1 } } \frac { 1 } { r _ { 3 } ( u _ { n - 2 } ) } \left[\cdots \left[\int_{T}^{u_{3}} \frac{1}{r_{n-1}\left(u_{2}\right)}\right.\right.\right. \\
& \left.\left.\left.\times\left[\int_{T}^{u_{2}} \frac{1}{r_{n}\left(u_{1}\right)} \Delta u_{1}\right]^{\frac{3}{2}} \Delta u_{2}\right] \cdots\right] \triangle u_{n-2}\right] \triangle u_{n-1} \\
& \geq \int_{2^{n-2} T}^{t} \frac{1}{u_{n-1}}\left[\int_{2^{n-3} T}^{u_{n-1}} \frac{1}{u_{n-2}}\right. \\
& \left.\times\left[\cdots\left[\int_{2 T}^{u_{3}}\left[\frac{1}{u_{2}^{\frac{1}{4}}} \int_{T}^{u_{2}} \frac{1}{u_{1}^{\frac{1}{2}}} \Delta u_{1}\right]^{\frac{3}{2}} \Delta u_{2}\right] \cdots\right] \Delta u_{n-2}\right] \Delta u_{n-1} \\
& \geq \int_{2^{n-2} T}^{t} \frac{1}{u_{n-1}}\left[\int_{2^{n-3} T}^{u_{n-1}} \frac{1}{u_{n-2}}\right. \\
& \left.\times\left[\cdots\left[\int_{2 T}^{u_{3}}\left[\frac{1}{u_{2}^{\frac{3}{4}}}\left(u_{2}-T\right)\right]^{\frac{3}{2}} \Delta u_{2}\right] \cdots\right] \triangle u_{n-2}\right] \triangle u_{n-1} \\
& \geq\left(\frac{1}{2}\right)^{n-\frac{1}{2}}\left(t-2^{n-2} T\right) \text {. }
\end{aligned}
$$

Pick $T_{*}>T>0$ such that

$$
\frac{1}{t^{\frac{1}{3}}} \geq \frac{1}{t^{\frac{1}{2}}} \geq \frac{1}{\left[\left(\frac{1}{2}\right)^{n-\frac{1}{2}}\left(t-2^{n-2} T\right)\right]^{\frac{2}{3}}} \quad \text { for } t \geq T_{*}
$$

Take $M(t)=t$ and $H(t, s)=1$ for $t>s \geq 2$ and $H(t, t)=0$ for $t \geq 2$, then

$$
h(t, s)=-\frac{1}{s^{\frac{2}{5}}} .
$$

Therefore we have that if $\rho>\frac{1}{\left(\frac{5}{3}\right)^{\frac{5}{3}}}$, then

$$
\begin{aligned}
& \limsup _{t \rightarrow \infty} \frac{1}{H(t, T)} \int_{T}^{t}\left[M(s) q(s) H(t, s)-\frac{h_{-}^{\gamma+1}(t, s) r_{1}^{\gamma}(s)}{(\gamma+1)^{\gamma+1} \vartheta_{2}^{\gamma}(s, T)}\right] \triangle s \\
& \quad=\limsup _{t \rightarrow \infty} \int_{T}^{t}\left[\frac{\rho}{s^{\frac{1}{3}}}-\frac{1}{\left(\frac{5}{3}\right)^{\frac{5}{3}}\left[\left(\frac{1}{2}\right)^{n-\frac{1}{2}}\left(s-2^{n-2} T\right)\right]^{\frac{2}{3}}}\right] \triangle s \\
& \quad \geq\left(\rho-\frac{1}{\left(\frac{5}{3}\right)^{\frac{5}{3}}}\right) \limsup _{t \rightarrow \infty} \int_{T_{*}}^{t} \frac{1}{s^{\frac{1}{2}}} \triangle s
\end{aligned}
$$




$$
\begin{aligned}
& =\left(\rho-\frac{1}{\left(\frac{5}{3}\right)^{\frac{5}{3}}}\right) \limsup _{t \rightarrow \infty} \frac{t^{\frac{1}{2}}-\left(T_{*}\right)^{\frac{1}{2}}}{2^{\frac{1}{2}}-1} \\
& =\infty
\end{aligned}
$$

Thus conditions $\left(\mathrm{H}_{3}\right)$, (2.1) and (3.24) are satisfied. By Theorem 3.2 every solution of Eq. (4.3) is either oscillatory or tends to zero if $\rho>\frac{1}{\left(\frac{5}{3}\right)^{\frac{5}{3}}}$. The proof is completed.

Example 4.3 Consider the following higher order dynamic equation:

$$
S_{n}^{\triangle}(t, x(t))+\frac{\rho}{t \sigma(t)} x^{\gamma}(\tau(t))=0
$$

on an arbitrary time scale $\mathbf{T}$, where $n \geq 2, \gamma \geq 1$ is the quotient of two odd positive integers, $\rho$ is a positive constant, $S_{k}(t, x(t))(0 \leq k \leq n)$ are as in Eq. (1.3) with $r_{n}(t)=1, r_{n-1}(t)=t^{\frac{1}{\gamma}}$, $r_{n-2}(t)=\cdots=r_{1}(t)=t$ and $\tau$ is defined as in $\left(\mathrm{H}_{4}\right)$. If $\rho>2^{(n-1) \gamma+1}$, then every solution of Eq. (4.5) is either oscillatory or tends to zero.

Proof Note that

$$
\begin{aligned}
& \int_{t_{0}}^{\infty}\left[\frac{1}{r_{n}(s)}\right]^{\frac{1}{\gamma}} \Delta s=\int_{t_{0}}^{\infty} \Delta s=\infty, \\
& \int_{t_{0}}^{\infty} \frac{1}{r_{n-1}(s)} \Delta s=\int_{t_{0}}^{\infty} \frac{1}{s^{\frac{1}{\gamma}}} \Delta s=\infty
\end{aligned}
$$

and

$$
\int_{t_{0}}^{\infty} \frac{1}{r_{i}(s)} \Delta s=\int_{t_{0}}^{\infty} \frac{1}{s} \Delta s=\infty \quad \text { for } 1 \leq i \leq n-2
$$

Pick that $t_{*} \geq t_{0}$ such that $\int_{t_{0}}^{t_{*}} \frac{1}{{ }_{s}^{\frac{1}{\gamma}}} \Delta s>0$, then

$$
\begin{aligned}
& \int_{t_{0}}^{\infty} \frac{1}{r_{n-1}(s)}\left\{\int_{s}^{\infty}\left[\frac{1}{r_{n}(u)} \int_{u}^{\infty} q(v) \Delta v\right]^{\frac{1}{\gamma}} \Delta u\right\} \Delta s \\
& \quad=\int_{t_{0}}^{\infty} \frac{1}{s^{\frac{1}{\gamma}}}\left\{\int_{s}^{\infty}\left[\int_{u}^{\infty} \frac{\rho}{v \sigma(v)} \Delta v\right]^{\frac{1}{\gamma}} \Delta u\right\} \Delta s \\
& \geq(\rho)^{\frac{1}{\gamma}} \int_{t_{0}}^{\infty} \frac{1}{s^{\frac{1}{\gamma}}}\left\{\int_{s}^{\infty} \frac{1}{u^{\frac{1}{\gamma}}} \Delta u\right\} \Delta s \\
& \geq(\rho)^{\frac{1}{\gamma}} \int_{t_{0}}^{t_{*}} \frac{1}{s^{\frac{1}{\gamma}}} \Delta s \int_{s_{*}}^{\infty} \frac{1}{u^{\frac{1}{\gamma}}} \Delta u \\
& =\infty .
\end{aligned}
$$

Using arguments similar to that of (4.4), it is easy to see that $\vartheta_{1}(t, T) \geq\left(\frac{1}{2}\right)^{n-1+\frac{1}{\gamma}}\left(t-2^{n-1} T\right)$. Therefore we have that if $\rho>2^{(n-1) \gamma+1}$, then

$$
\limsup _{t \rightarrow \infty} \vartheta_{1}^{\gamma}(t, T) \int_{t}^{\infty} q(s) \Delta s \geq \rho\left(\frac{1}{2}\right)^{(n-1) \gamma+1} \limsup _{t \rightarrow \infty} \frac{\left(t-2^{n-1} T\right)^{\gamma}}{t} \geq \rho\left(\frac{1}{2}\right)^{(n-1) \gamma+1}>1 .
$$


Thus conditions $\left(\mathrm{H}_{3}\right),(2.1)$ and (3.34) are satisfied. By Theorem 3.3 every solution of Eq. (4.5) is either oscillatory or tends to zero if $\rho>2^{(n-1) \gamma+1}$. The proof is completed.

Example 4.4 Consider the following third-order dynamic equation:

$$
\left[\left(t x^{\triangle}(t)\right)^{\triangle}\right]^{\triangle}+\frac{3 t\left[\left(4 t^{2}+18 t+19\right)(t+1)+\left(4 t^{2}+10 t+5\right)(t+3)\right]}{(t+1)(t+2)(t+3)} x(3 t)=0
$$

on $\mathbf{N}=\{1,2,3, \ldots\}$, where $n=2, \gamma=1, r_{2}(t)=1, r_{1}(t)=t$ and $\tau(t)=3 t$ for any $t \in \mathbf{N}$. It is easy to see that conditions $\left(\mathrm{H}_{1}\right)-\left(\mathrm{H}_{5}\right)$ are satisfied and $x(t)=\frac{(-1)^{t}}{t}$ is an oscillatory solution of Eq. (4.6), which tends to zero as $t \rightarrow \infty$.

\section{Competing interests}

The authors declare that they have no competing interests.

\section{Authors' contributions}

All authors contributed equally to the manuscript and typed, read, and approved the final manuscript.

\section{Author details}

${ }^{1}$ College of Mathematics and Information Science, Guangxi University, Nanning, Guangxi 530004, P.R. China.

${ }^{2}$ Department of Mathematics, Guangxi College of Finance and Economics, Nanning, Guangxi 530003, P.R. China.

\section{Acknowledgements}

The authors express their sincere gratitude to the referees for their valuable suggestions and comments. This paper is project is supported by NNSF of China (11261005) and NSF of Guangxi (2011GXNSFA018135, 2012GXNSFDA276040) and SF of ED of Guangxi (2013ZD061).

Received: 7 April 2013 Accepted: 1 August 2013 Published: 20 August 2013

\section{References}

1. Hilger, S: Analysis on measure chains - a unified approach to continuous and discrete calculus. Results Math. 18, 18-56 (1990)

2. Kac, V, Chueng, P: Quantum Calculus. Universitext (2002)

3. Agarwal, RP: Difference Equations and Inequalities. Theory, Methods and Applications, 2nd edn. Dekker, New York (2000)

4. Agarwal, RP, Bohner, M, Saker, SH: Oscillation of second order delay dynamic equations. Can. Appl. Math. Q. 13, 1-17 (2005)

5. Bohner, M, Karpuz, B, Ocalan, O: Iterated oscillation criteria for delay dynamic equations of first order. Adv. Differ. Equ. 2008, Article ID 458687 (2008)

6. Bohner, M, Peterson, A: Advances in Dynamic Equations on Time Scales. Birkhäuser, Boston (2003)

7. Bohner, M, Saker, SH: Oscillation of second order nonlinear dynamic equations on time scales. Rocky Mt. J. Math. 34, 1239-1254 (2004)

8. Erbe, L: Oscillation results for second-order linear equations on a time scale. J. Differ. Equ. Appl. 8, 1061-1071 (2002)

9. Erbe, L, Peterson, A, Saker, SH: Oscillation criteria for second-order nonlinear delay dynamic equations. J. Math. Anal. Appl. 333, 505-522 (2007)

10. Grace, SR, Agarwal, RP, Kaymakcalan, B, Sae-Jie, W: On the oscillation of certain second order nonlinear dynamic equations. Math. Comput. Model. 50, 273-286 (2009)

11. Hardy, GH, Littlewood, JE, Polya, G: Inequalities, 2nd edn. Cambridge University Press, Cambridge (1988)

12. Hassan, TS: Oscillation criteria for half-linear dynamic equations on time scales. J. Math. Anal. Appl. 345, 176-185 (2008)

13. Han, Z, Sun, S, Shi, B: Oscillation criteria for a class of second-order Emden-Fowler delay dynamic equations on time scales. J. Math. Anal. Appl. 334, 847-858 (2007)

14. Han, Z, Shi, B, Sun, S: Oscillation criteria for second-order delay dynamic equations on time scales. Adv. Differ. Equ. 2007, Article ID 70730 (2007)

15. Sun, $\mathrm{T}, \mathrm{Xi}, \mathrm{H}, \mathrm{Peng}, \mathrm{X}$ : Asymptotic behavior of solutions of higher-order dynamic equations on time scales. Adv. Differ. Equ. 2011, Article ID 237219 (2011)

16. Sun, $T, X i, H$, Peng, X, Yu, W: Nonoscillatory solutions for higher-order neutral dynamic equations on time scales. Abstr. Appl. Anal. 2010, Article ID 428963 (2010)

17. Sun, $T, X i, H, Y u, W$ : Asymptotic behaviors of higher order nonlinear dynamic equations on time scales. J. Appl. Math Comput. 37, 177-192 (2011)

18. Wang, Y, Xu, Z: Asymptotic properties of solutions of certain third-order dynamic equations. J. Comput. Appl. Math. 236, 2354-2366 (2012)

19. Erbe, L, Peterson, A, Saker, SH: Asymptotic behavior of solutions of a third-order nonlinear dynamic equation on time scales. J. Comput. Appl. Math. 181, 92-102 (2005) 
20. Erbe, L, Peterson, A, Saker, SH: Oscillation and asymptotic behavior a third-order nonlinear dynamic equation. Can. Appl. Math. Q. 14, 129-147 (2006)

21. Erbe, L, Peterson, A, Saker, SH: Hille and Nehari type criteria for third order dynamic equations. J. Math. Anal. Appl. 329, 112-131 (2007)

22. Hassan, TS: Oscillation of third order nonlinear delay dynamic equations on time scales. Math. Comput. Model. 49 1573-1586 (2009)

23. Hassan, TS: Kamenev-type oscillation criteria for second order nonlinear dynamic equations on time scales. Appl. Math. Comput. 217, 5285-5297 (2011)

24. Sun, $T, Y u, W, X i, H$ : Oscillatory behavior and comparison for higher order nonlinear dynamic equations on time scales. J. Appl. Math. Inf. 30, 289-304 (2012)

25. Bohner, M, Peterson, A: Dynamic Equations on Time Scales: An Introduction with Applications. Birkhäuser, Boston (2001)

doi:10.1186/1687-1847-2013-248

Cite this article as: Wu et al.: Kamenev-type oscillation criteria for higher-order nonlinear dynamic equations on time scales. Advances in Difference Equations 2013 2013:248.

\section{Submit your manuscript to a SpringerOpen ${ }^{\circ}$ journal and benefit from:}

- Convenient online submission

- Rigorous peer review

- Immediate publication on acceptance

Open access: articles freely available online

- High visibility within the field

- Retaining the copyright to your article 